\title{
Analysis of Longitudinal Mechanical Properties of Buried Suspended Pipeline Resisting Collapse
}

\author{
Yujian Lin, Yanping Lv $\mathbb{D}$, Xu Liu, and Fuquan Chen \\ College of Civil Engineering, Fuzhou University, No. 2 Xueyuan Road, University Town, Fuzhou 350116, China \\ Correspondence should be addressed to Yanping Lv; lvanna@fzu.edu.cn
}

Received 17 January 2019; Revised 5 March 2019; Accepted 25 March 2019; Published 18 April 2019

Academic Editor: Flavio Stochino

Copyright (c) 2019 Yujian Lin et al. This is an open access article distributed under the Creative Commons Attribution License, which permits unrestricted use, distribution, and reproduction in any medium, provided the original work is properly cited.

\begin{abstract}
Ground subsidence caused by collapse leads to suspension or subsidence of the pipe, resulting in the stress concentration. It is a significant factor being a threat to safe operation of the pipeline. Based on Pasternak's beam-on-elastic-foundation theory, this study presents a mechanical model of interaction between the buried suspended pipeline and the soil, which considers the effect of the beam width. The pipeline was modeled as a buried part and a suspended part. According to the compatibility conditions of suspended and nonsuspended parts, this paper solved differential equations describing the deflection of pipeline and derived the formulas of deflection and internal force. This solution involves whole cases of the Pasternak model. Connecting to the pipeline operation status, the formulas of piping stress were deduced by piping stress analysis. Meanwhile, piping stress analysis can define the scope of application of the model. Compared with the measured values and results of the Winkler model, the calculated results of the Pasternak model are more accurate and suitable for predicting the behavior of the pipeline in the limit state. In addition, according to normalized analysis of main affecting factors which comprise the pipe material, pipe dimension, pipe depth, and length of collapse, it was also found that the length of collapse dominates the stability of the pipeline.
\end{abstract}

\section{Introduction}

The buried pipelines have been widely used in urban constructions. The plastic buried pipeline attracts increasing attention compared with the metal and the cement buried pipelines due to advantages of light weight, cost-effectiveness, easy installation, and good flexibility. In some areas, overpumping the underground water induced the occurrence of the soil cave, which may cause serious ground collapse. The damage of the ground collapse to the pipeline is that the pipeline may be bent, sunk, or suspended and stress concentration occurs in some parts of the pipeline. If the stress state exceeds the limit strength, the pipeline will rupture. Therefore, it is necessary to predict the displacement and stress of the pipeline suffering the soil collapse to meet the needs of the pipelines safety operation.

Many studies have been carried out on pipelines to investigate the induced displacements and stresses under various conditions. Tanabe and Takada [1] dealt with modeling of the buried pipeline and proposed the corresponding design formula by using the elastic ground beam theory. Gao [2] simulated the deformations of the pipelines located in settlement and nonsettlement zones by using the cubic curve and the beam-on-elastic-foundation theory, respectively. In addition, Gao [2] also calculated the internal force acting on the junction of the settlement and nonsettlement parts. Using Winkler's beam-on-elastic-foundation theory, Chen and Zhang [3] proposed equations to estimate the stress distribution and the failure length of the pipeline buried in clay by taking the effects of the self-weight of the pipeline, the internal fluid pressure, and the cohesion of the clay into account.

Wang and Yao [4] proposed equations to calculate the deflection and internal force of pipelines using the compatibility of deformations of buried and suspended parts of the pipeline. In addition, Kouretzis et al. [5] investigated the internal force and the vertical displacement variations of the pipeline under permanent ground settlement by taking the nonlinearity of the pipeline material into consideration. Ma et al. [6] investigated the nonlinear response of a beam on elastic foundation subjected to the harmonic excitation and studied the effect of soil-structure interaction on the primary resonance of the beam. Cao et al. [7] conducted model tests to study the effects of the shallowly buried 
pipe on the permanent deformation of the road surface under the cyclic traffic loading. Also, they set up a mechanisticempirical model to estimate the permanent deformation of the road surface induced by the shallowly buried pipe under cyclic loading. Luo et al. [8] conducted numerical simulations to investigate the piping stress-settlement relationship of the PE pipeline under foundation settlement and the effect of the transition section length on the pipeline yielding.

In addition, many studies were carried out on the buried pipeline to calculate the stress and vertical displacement of the pipeline due to the ground subsidence using Winkler's beamon-elastic-foundation theory. Kim et al. [9] used Winkler's beam-on-elastic-foundation theory to analyze the maximum pipeline stress in three different subsidence cases and verified it by using finite element calculation software. Yu et al. [10] proposed the calculation formula for the influence of tunnel excavation settlement on the pipeline, based on the Winkler foundation beam model. And the results were compared with the existing experimental results. Iimura [11] derived the stress calculation formula of the buried part, the exposed part, and the intermediate part. Peng and Luo [12] and Luo et al. [13] analyzed the stress state of the thin-walled pipe under subsidence and obtained the stress and strain calculation formula under the same deformation of the pipe and ground.

In previous studies, the Winkler model was widely used in theoretical analysis and practical engineering of pipelines. However, the Winkler model assumes that the pipeline is supported by an array of discrete springs representing the soil, which neglects the soil continuity and the soil shearing resistance because each soil spring behaves independently. Pasternak's beam-on-elastic-foundation theory (also called the Pasternak model) can overcome those shortcomings. The Pasternak model is called the "two-parameter model" as it includes the coefficient of the subgrade reaction and shear modules of the soil. Tanahashi [14] presented a set of equations to estimate the displacements and stress on an infinitely long Bernoulli-Euler beam using the Pasternak model.

In this paper, the Pasternak model was employed. And a new mechanical model of the pipeline in the subsidence area was constructed by considering the three-dimensional influence of beam width on the deformation of the pipeline. The calculation formula of the stress, deflection, and internal force of the pipeline under the influence of the collapse effect were obtained.

\section{Calculation Model}

There is a straight continuous pipeline crossing an area where a soil cave is developed (Figure 1). The origin is taken at the intersection of the vertical center line of the soil cave and the horizontal axis of the pipeline prior to deformation. The coordinates $(x, y)$ represent the length and the vertical displacement of the pipeline, respectively. Since the deformed pipeline is symmetric with respect to the ordinate $y$, a half of the pipeline is only analyzed.

2.1. Calculation Model for the Buried Pipeline. As shown in Figure 1, the coordinate of the pipeline is constructed. To simplify the calculation, the pipeline is segmented into two

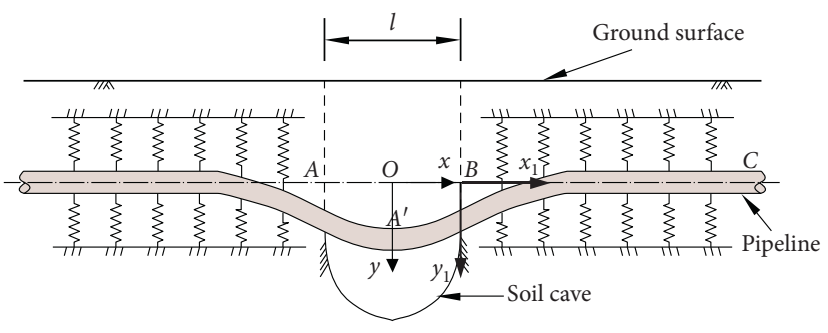

Figure 1: Calculation model.

parts: the suspended part (segment $A^{\prime} B$ ) and the buried part (segment $B C$ ). The buried part $B C$ is analyzed by employing the beam-on-elastic-foundation theory. The influence of the suspended segment $A B$ on the pipeline section at point $B$ can be modeled by an equivalent shear force $P$ and an equivalent moment $M$ acting at point $B$. In addition, it can be also seen from Figure 1 that segment $B C$ is also subjected to vertical forces induced by the earth pressure due to the upper soil, the self-weight of the pipeline, and the internal fluid weight.

The vertical displacement $y_{1}$ of a half infinitely long beam on the Pasternak model in the two-dimensional plain strain condition can be expressed as follows [14-16]:

$$
\begin{gathered}
E I \frac{d^{4} y_{1}}{d x_{1}^{4}}=b q\left(x_{1}\right)-P\left(x_{1}\right), \\
P\left(x_{1}\right)=k y_{1} b^{*}-G_{\mathrm{P}} b^{*} y_{1}^{\prime \prime}, \\
b^{*}=b\left(1+\frac{\sqrt{G_{\mathrm{P}}}}{b^{2} k}\right),
\end{gathered}
$$

where $E I$ is the pipeline bending stiffness, $P\left(x_{1}\right)$ is the subgrade reaction force acting on the beam, $q\left(x_{1}\right)$ is the vertical load acting on the beam, $k$ is the coefficient of the subgrade reaction, $G_{\mathrm{P}}$ is the shear modules of the soil, and $b^{*}$ is the effective width of the beam.

Substituting Equations (2) and (3) into Equation (1), we obtain

$$
\frac{d^{4} y_{1}}{d x_{1}^{4}}-4 \rho \alpha^{2} y_{1}^{\prime \prime}+4 \alpha^{4} y_{1}=\frac{b q\left(x_{1}\right)}{E I},
$$

where

$$
\begin{aligned}
& \alpha=\sqrt[4]{\frac{k b^{*}}{4 E I}}, \\
& \rho=\frac{G_{\mathrm{P}}}{2 \times \sqrt{k E I / b^{*}} .} .
\end{aligned}
$$

The characteristic equation of the homogeneous differential equation of Equation (4) is

$$
m^{4}-4 \rho \alpha^{2} m^{2}+4 \alpha^{4}=0 .
$$

Equation (6) yields

$$
m_{1,2,3,4}= \pm \alpha \sqrt{2\left(\rho \pm \sqrt{\rho^{2}-1}\right)} .
$$

There are three possible general solutions of Equation (4), depending on the value of $\rho(\rho>1, \rho=1$, or $\rho<1)$. When $\rho<1$, the general solution of Equation (4) is 


$$
\begin{aligned}
y_{1}= & \left(A_{1} e^{-\varphi_{1} x}+A_{2} e^{\varphi_{1} x}\right) \cos \varphi_{2} x \\
& +\left(A_{3} e^{-\varphi_{1} x}+A_{4} e^{\varphi_{1} x}\right) \sin \varphi_{2} x,
\end{aligned}
$$

where

$$
\begin{aligned}
& \varphi_{1}=\alpha \sqrt{1+\rho}, \\
& \varphi_{2}=\alpha \sqrt{1-\rho} .
\end{aligned}
$$

When $\rho=1$, the general solution of Equation (4) is

$$
y_{1}=e^{-\varphi_{3} x}\left(B_{1}+B_{2} x\right)+e^{\varphi_{3} x}\left(B_{3}+B_{4} x\right),
$$

where

$$
\varphi_{3}=\sqrt{2} \alpha
$$

When $\rho>1$, Equation (4) yields

$$
\begin{aligned}
y_{1}= & \left(C_{1} e^{-\varphi_{1} x}+C_{2} e^{\varphi_{1} x}\right) \cosh \varphi_{4} x \\
& +\left(C_{3} e^{-\varphi_{1} x}+C_{4} e^{\varphi_{1} x}\right) \sinh \varphi_{4} x,
\end{aligned}
$$

where

$$
\begin{aligned}
& \varphi_{1}=\alpha \sqrt{1+\rho}, \\
& \varphi_{2}=\alpha \sqrt{\rho-1} .
\end{aligned}
$$

As shown in Figure 1, point $B$ is subjected to an equivalent vertical concentrated load $P$ and an equivalent concentrated moment $m$. In addition, an uniform load $q_{1}$ is applied on the buried pipeline. Therefore, the weighted residual method can be used for obtaining the solution of Equation (4). Solutions under the vertical concentrated load $P$, the concentrated moment $m$, and the uniform load $q_{1}$ are first calculated separately. Then, the solution of Equation (4) can be obtained by superposition of the solutions under $P, m$, and $q_{1}$. In this study, the case of $\rho<1$ was used for calculation.

(1) Solution under vertical concentrated load $P$ : the general solution of the displacement of the pipeline is

$$
\begin{aligned}
\tilde{y}_{\mathrm{p}}= & \left(A_{1} e^{-\varphi_{1} x}+A_{2} e^{\varphi_{1} x}\right) \cos \varphi_{2} x \\
& +\left(A_{3} e^{-\varphi_{1} x}+A_{4} e^{\varphi_{1} x}\right) \sin \varphi_{2} x,
\end{aligned}
$$

and the boundary conditions of the trial function is

$$
\tilde{y}_{\mathrm{p}}=0 \text {, }
$$

when $x=\infty$. When $x=0$, the boundary conditions of the trial function are

$$
\left.\begin{array}{l}
-E I \frac{d^{2} \widetilde{y}_{\mathrm{p}}}{d x^{2}}=0, \\
-E I \frac{d^{3} \widetilde{y}_{\mathrm{p}}}{d x^{3}}=-P .
\end{array}\right\}
$$

The residuals can be eliminated by using the collocation method and can be expressed as

$$
\left.\begin{array}{l}
R_{1}=E I\left(A_{1} \varphi_{2}^{2}+2 A_{3} \varphi_{1} \varphi_{2}-A_{1} \varphi_{1}^{2}\right)=0, \\
R_{2}=P-E I\left(A_{1} \varphi_{1}^{3}-3 A_{3} \varphi_{1}^{2} \varphi_{2}-3 A_{1} \varphi_{1} \varphi_{2}^{2}+A_{3} \varphi_{2}^{3}\right)=0 .
\end{array}\right\}
$$

Therefore, we get

$\tilde{y}_{\mathrm{p}}=\frac{P}{E I} e^{-\varphi_{1} x}\left[\frac{2 \varphi_{1} \cos \varphi_{2} x}{\varphi_{1}^{4}+2 \varphi_{1}^{2} \varphi_{2}^{2}+\varphi_{2}^{4}}+\frac{\left(\varphi_{1}^{2}-\varphi_{2}^{2}\right) \sin \varphi_{2} x}{\varphi_{1}^{4} \varphi_{2}+2 \varphi_{1}^{2} \varphi_{2}^{3}+\varphi_{2}^{5}}\right]$.

(2) Solution under moment $m$ : the general solution of the displacement can be also expressed using Equation (11). The corresponding boundary condition is

$$
\tilde{y}_{m}=0 \text {, }
$$

when $x=\infty$. When $x=0$, the corresponding boundary conditions are

$$
\left.\begin{array}{l}
-E I \frac{d^{2} \widetilde{y}_{m}}{d x^{2}}=m, \\
-E I \frac{d^{3} \widetilde{y}_{m}}{d x^{3}}=0 .
\end{array}\right\}
$$

The residuals can be eliminated by using the collocation method and can be expressed as

$$
\left.\begin{array}{l}
R_{1}=m-E I\left(A_{1} \varphi_{2}^{2}+2 A_{3} \varphi_{1} \varphi_{2}-A_{1} \varphi_{1}^{2}\right)=0, \\
R_{2}=E I\left(A_{1} \varphi_{1}^{3}-3 A_{3} \varphi_{1}^{2} \varphi_{2}-3 A_{1} \varphi_{1} \varphi_{2}^{2}+A_{3} \varphi_{2}^{3}\right)=0,
\end{array}\right\}
$$

and then we get

$$
\tilde{y}_{m}=\frac{m}{E I} e^{-\varphi_{1} x}\left[\frac{\left(\varphi_{2}^{2}-3 \varphi_{1}^{2}\right) \cos \varphi_{2} x}{\varphi_{1}^{4}+2 \varphi_{1}^{2} \varphi_{2}^{2}+\varphi_{2}^{4}}+\frac{\left(3 \varphi_{1} \varphi_{2}^{2}-\varphi_{1}^{3}\right) \sin \varphi_{2} x}{\varphi_{1}^{4} \varphi_{2}+2 \varphi_{1}^{2} \varphi_{2}^{3}+\varphi_{2}^{5}}\right] \text {. }
$$

(3) Solution under uniform load $q_{1}$ : the general solution of the displacement of the pipeline is of the following form:

$$
\tilde{y}_{q}=a_{0}+a_{1} x_{1}+a_{2} x_{1}^{2}+a_{3} x_{1}^{3}+\ldots+a_{8} x_{1}^{8} .
$$

Substituting Equation (18) into Equation (4) gives

$$
\begin{aligned}
& a_{4}=\frac{q_{1} b}{24 E I}-\frac{1}{6} \alpha^{4} a_{0}+\frac{1}{3} \rho \alpha^{2} a_{2}, \\
& a_{i}=\frac{4 \alpha^{2} \rho}{i(i-1)} a_{i-2}+\frac{4 \alpha^{4}}{i(i-1)(i-2)(i-3)(i-4)} a_{i-4}, \quad 5 \leq i \leq 8 .
\end{aligned}
$$

In addition, the displacement $y_{\mathrm{q}}$ satisfies the following boundary conditions:

$$
\begin{array}{r}
\left.\frac{d y_{q}}{d x_{1}}\right|_{x_{1}=L / 2}=0, \\
-\left.E I \frac{d^{3} y_{q}}{d x_{1}^{3}}\right|_{x_{1}=L / 2}=0, \\
-\left.E I \frac{d^{2} y_{q}}{d x_{1}^{2}}\right|_{x_{1}=0}=0 .
\end{array}
$$


Thus, it can be concluded that $a_{0}=q_{1} b / 4 E I \alpha^{4}$ and $a_{1}=a_{2}=a_{3}=a_{4}=a_{4}=a_{5}=a_{6}=a_{7}=a_{8}=0$. Therefore, Equation (18) can be written as

$$
\tilde{y}_{q}=\frac{q_{1}}{k\left[1+\sqrt{\left(G_{P} / k\right)} / b\right]} \text {. }
$$

Therefore, Equation (4) can be solved completely, and five mechanical quantities (vertical displacement $y$, rotation angle $\theta$, moment $M$, shear force $Q$, and load intensity $q$ ) can be obtained by superposition of Equations (14), (17), and (21) and by using the coordinate transformation:

$$
\left[\begin{array}{l}
y \\
\theta \\
Q
\end{array}\right]=\left[\begin{array}{c}
\left.\frac{e^{-\varphi_{1}(x-l / 2)}}{E I}\left[\frac{\left(2 P \varphi_{1}+m \varphi_{2}^{2}-3 m \varphi_{1}^{2}\right) \cos \left(\varphi_{2} x-\varphi_{2} l / 2\right)}{\varphi_{1}^{4}+2 \varphi_{1}^{2} \varphi_{2}^{2}+\varphi_{2}^{4}}+\frac{\left(P \varphi_{1}^{2}-P \varphi_{2}^{2}+3 m \varphi_{1} \varphi_{2}^{2}-m \varphi_{1}^{3}\right) \sin \left(\varphi_{2} x-\varphi_{2} l / 2\right)}{\varphi_{1}^{4} \varphi_{2}+2 \varphi_{1}^{2} \varphi_{2}^{3}+\varphi_{2}^{5}}\right]+\frac{b q_{1}}{k\left(b+\sqrt{\left.G_{\mathrm{p}} / k\right)}\right.}\right] \\
-\frac{e^{-\varphi_{1}(x-l / 2)}}{E I}\left[\frac{\left(m \varphi_{2}^{2}-m \varphi_{1}^{2}+P \varphi_{1}\right) \sin \left(\varphi_{2} x-\varphi_{2} l / 2\right)+\left(P \varphi_{2}-2 m \varphi_{1} \varphi_{2}\right) \cos \left(\varphi_{2} x-\varphi_{2} l / 2\right)}{\varphi_{2}\left(\varphi_{1}^{2}+\varphi_{2}^{2}\right)}\right] \\
\left.\frac{e^{-\varphi_{1}(x-l / 2)}}{\varphi_{2}}\left[m \varphi_{2} \cos \left(\varphi_{2} x-\varphi_{2} \frac{l}{2}\right)-P \sin \left(\varphi_{2} x-\varphi_{2} \frac{l}{2}\right)+m \varphi_{1} \sin \left(\varphi_{2} x-\varphi_{2} \frac{l}{2}\right)\right]\right] \\
-\frac{e^{-\varphi_{1}(x-l / 2)}}{\varphi_{2}}\left[m \varphi_{1}^{2} \sin \left(\varphi_{2} x-\varphi_{2} \frac{l}{2}\right)+m \varphi_{2}^{2} \sin \left(\varphi_{2} x-\varphi_{2} \frac{l}{2}\right)-P \varphi_{1} \sin \left(\varphi_{2} x-\varphi_{2} \frac{l}{2}\right)+P \varphi_{2} \cos \left(\varphi_{2} x-\varphi_{2} \frac{l}{2}\right)\right] \\
\frac{e^{-\varphi_{1}(x-l / 2)}}{\varphi_{2}}\left[\left(P \varphi_{2}^{2}-P \varphi_{1}^{2}+m \varphi_{1}^{3}+m \varphi_{1} \varphi_{2}^{2}\right) \sin \left(\varphi_{2} x-\varphi_{2} \frac{l}{2}\right)+\left(2 P \varphi_{1} \varphi_{2}-m \varphi_{2}^{3}-m \varphi_{1}^{2} \varphi_{2}\right) \cos \left(\varphi_{2} x-\varphi_{2} \frac{l}{2}\right)\right]
\end{array}\right] .
$$

In a similar manner, the solutions of Equation (4) for the cases of $\rho=1$ and $\rho>1$ can be obtained and given in Equations (23) and (24), respectively:

$$
\left[\begin{array}{l}
y \\
\theta \\
Q
\end{array}\right]=\left[\begin{array}{c}
\frac{e^{-\varphi_{1}(x-l / 2)}}{\varphi_{1}^{3} E I}\left[2 P+P \varphi_{1}\left(x-\frac{l}{2}\right)+3 m \varphi_{1}+m \varphi_{1}^{2}\left(x-\frac{l}{2}\right)\right]+\frac{b q_{1}}{k\left(b+\sqrt{G_{\mathrm{P}} / k}\right)} \\
-\frac{e^{-\varphi_{1}(x-l / 2)}}{\varphi_{1}^{2} E I}\left[P+2 m \varphi_{1}+m \varphi_{1}^{2}\left(x-\frac{l}{2}\right)+P \varphi_{1}\left(x-\frac{l}{2}\right)\right] \\
-e^{-\varphi_{1}(x-l / 2)}\left[m+P\left(x-\frac{l}{2}\right)+m \varphi_{1}\left(x-\frac{l}{2}\right)\right] \\
e^{-\varphi_{1}(x-l / 2)}\left[m \varphi_{1}^{2}\left(x-\frac{l}{2}\right)+P \varphi_{1}\left(x-\frac{l}{2}\right)-P\right] \\
\varphi_{1} e^{-\varphi_{1}(x-l / 2)}\left[2 P+m \varphi_{1}-m \varphi_{1}^{2}\left(x-\frac{l}{2}\right)-P \varphi_{1}\left(x-\frac{l}{2}\right)\right]
\end{array}\right],
$$




$$
\left[\begin{array}{c}
y \\
\theta \\
M \\
Q \\
q
\end{array}\right]=\left[\begin{array}{c}
\left.\frac{e^{-\varphi_{1}(x-l / 2)}}{E I}\left[\frac{\left(2 P \varphi_{1} \varphi_{2}-3 m \varphi_{1}^{2} \varphi_{2}-m \varphi_{2}^{3}\right) \cosh \left(\varphi_{2} x-\varphi_{2} l / 2\right)+\left(P \varphi_{1}^{2}+P \varphi_{2}^{2}-m \varphi_{1}^{3}-3 m \varphi_{1} \varphi_{2}^{2}\right) \sinh \left(\varphi_{2} x-\varphi_{2} l / 2\right)}{\varphi_{1}^{4} \varphi_{2}-2 \varphi_{1}^{2} \varphi_{2}^{3}+\varphi_{2}^{5}}\right]+\frac{b q_{1}}{k\left(b+\sqrt{\left.G_{\mathrm{P}} / k\right)}\right.}\right] \\
\frac{e^{-\varphi_{1}(x-l / 2)}}{\left(\varphi_{1}^{2}-\varphi_{2}^{2}\right) \varphi_{2} E I}\left[\left(m \varphi_{1}^{2}+m \varphi_{2}^{2}-P \varphi_{1}\right) \sinh \left(\varphi_{2} x-\varphi_{2} \frac{l}{2}\right)+\left(2 m \varphi_{1} \varphi_{2}-P \varphi_{2}\right) \cosh \left(\varphi_{2} x-\varphi_{2} \frac{l}{2}\right)\right] \\
\frac{e^{-\varphi_{1}(x-l / 2)}}{\varphi_{2}}\left[\left(m \varphi_{1}-P\right) \sinh \left(\varphi_{2} x-\varphi_{2} \frac{l}{2}\right)+m \varphi_{2} \cosh \left(\varphi_{2} x-\varphi_{2} \frac{l}{2}\right)\right] \\
-\frac{e^{-\varphi_{1}(x-l / 2)}}{\varphi_{2}}\left[\left(m \varphi_{1}^{2}-m \varphi_{2}^{2}-P \varphi_{1}\right) \sinh \left(\varphi_{2} x-\varphi_{2} \frac{l}{2}\right)+P \varphi_{2} \cosh \left(\varphi_{2} x-\varphi_{2} \frac{l}{2}\right)\right] \\
-\frac{e^{-\varphi_{1}(x-l / 2)}}{\varphi_{2}}\left[\left(P \varphi_{1}^{2}+P \varphi_{2}^{2}-m \varphi_{1}^{3}+m \varphi_{1} \varphi_{2}^{2}\right) \sinh \left(\varphi_{2} x-\varphi_{2} \frac{l}{2}\right)+\left(m \varphi_{1}^{2} \varphi_{2}-m \varphi_{2}^{3}-2 P \varphi_{1} \varphi_{2}\right) \cosh \left(\varphi_{2} x-\varphi_{2} \frac{l}{2}\right)\right.
\end{array}\right] .
$$

2.2. Calculation Model for the Suspended Pipeline. The suspended pipeline can be simplified into a simply supported beam subjected to moments on both ends (Figure 2). Local coordinates are constructed, and the origin is located at point $A$.

As shown in Figure 2, the suspended pipeline is subjected to moments on both ends and the uniform load $q_{2}$ that is induced by the self-weight of the pipeline and self-weight of the upper soil and the internal fluid weight. Therefore, the five mechanical quantities can be obtained by the principle of superposition.

The formula for obtaining the vertical displacement of the pipeline subjected to moment acting on the left end of the pipeline can be expressed as

$$
y_{m L}=\frac{m x_{2}}{6 l E I}\left(l-x_{2}\right)\left(2 l-x_{2}\right)
$$

The formula for obtaining the vertical displacement of the pipeline subjected to moment acting on the right end can be expressed as

$$
y_{m R}=\frac{m x_{2}}{6 l E I}\left(l^{2}-x_{2}^{2}\right) .
$$

In addition, the formula for estimating the pipeline vertical displacement subjected to uniform load can be expressed as

$$
y_{q}=\frac{q_{2} b x_{2}}{24 E I}\left(l^{3}-2 l x_{2}^{2}+x_{2}^{3}\right)
$$

Therefore, the five mechanical quantities (vertical displacement $y$, rotation angle $\theta$, moment $M$, shear force $Q$, and load intensity $q$ ) of the suspended pipeline can be obtained by superposition of Equations (25)-(27) and by using the coordinate transformation:

$$
\left[\begin{array}{l}
y \\
\theta \\
M \\
Q \\
q
\end{array}\right]=\left[\begin{array}{c}
\frac{2 \beta(P-m \beta)+b q_{1}}{k}+\frac{\left(l^{2}-4 x^{2}\right)\left(5 q_{2} b l^{2}-4 q_{2} b x^{2}+48 m\right)}{384 E I} \\
\frac{\left(4 q_{2} b x^{3}-3 q_{2} b x l^{2}-24 m x\right)}{24 E I} \\
m+\frac{q_{2} b l^{2}}{8}-\frac{q_{2} b x^{2}}{2} \\
-q_{2} b x \\
-q_{2} b
\end{array}\right]
$$

2.3. Calculation Model for the Entire Pipeline. To satisfy the displacement compatibility, displacement and rotation angle of the suspended pipeline at point $B$ should be equal to those of the buried pipeline. Therefore, for the cases of $\rho<1, \rho=1$, and $\rho>1$, we obtain 


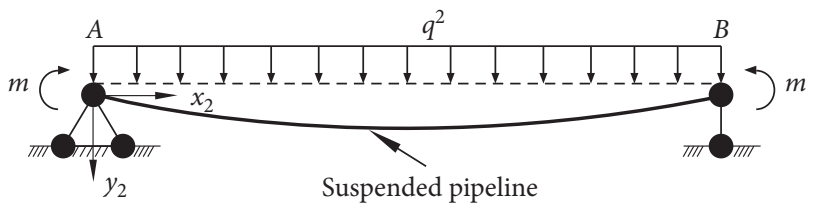

Figure 2: Calculation model for the suspended pipeline.

$$
\left.\begin{array}{rr}
\frac{12 m l+q_{2} b l^{3}}{24 E I}=\frac{P \varphi_{2}-2 m \varphi_{1} \varphi_{2}}{\varphi_{2}\left(\varphi_{1}^{2}+\varphi_{2}^{2}\right) E I}, & \rho<1, \\
\frac{12 m l+q_{2} b l^{3}}{24 E I}=\frac{P+2 m \varphi_{1}}{\varphi_{1}^{2} E I}, & \rho=1, \\
\frac{12 m l+q_{2} b l^{3}}{24 E I}=\frac{P \varphi_{2}-2 m \varphi_{1} \varphi_{2}}{\varphi_{2}\left(\varphi_{1}^{2}-\varphi_{2}^{2}\right) E I}, & \rho>1 .
\end{array}\right\}
$$

Since $P=q_{2} l / 2$, Equation (29) yields

$$
\left.\begin{array}{cc}
m=\frac{12 q_{2} b l-q_{2} b \varphi_{2}^{2} l^{3}-q_{2} b \varphi_{1}^{2} l^{3}}{12 \varphi_{1}^{2} l+48 \varphi_{1}+12 \varphi_{2}^{2} l}, & \rho<1, \\
m=\frac{\varphi_{1}^{2} q_{2} b l^{3}-12 q_{2} b l}{48 \varphi_{1}-12 \varphi_{1}^{2} l}, & \rho=1, \\
m=\frac{12 q_{2} b l+q_{2} b \varphi_{2}^{2} l^{3}-q_{2} b \varphi_{1}^{2} l^{3}}{12 \varphi_{1}^{2} l+48 \varphi_{1}-12 \varphi_{2}^{2} l}, & \rho>1 .
\end{array}\right\}
$$

\section{Stresses of Pipelines}

When the pipeline that crosses areas suffering soil collapse, the stresses of the pipeline consist of the tangential and axial stresses induced by internal fluid pressure, the stress induced by the change in temperature, and the stresses induced by pipeline bending. These stresses can be calculated as follows.

3.1. Stress Induced by Internal Fluid Pressure. As shown in Figure 3 , the tangential stress $\sigma_{\mathrm{pt}}$ induced by the internal fluid pressure can be calculated by Press [17]:

$$
\begin{aligned}
t \sigma_{\mathrm{pt}} & =\int_{0}^{\pi} P(D-2 t) \sin \alpha d \alpha, \\
\sigma_{\mathrm{pt}} & =\frac{P(D-2 t)}{2 t} .
\end{aligned}
$$

The axial stress $\sigma_{\mathrm{pa}}$ induced by the internal fluid pressure can be obtained by

$$
\sigma_{\mathrm{pa}}=\mu \sigma_{\mathrm{pt}}=\frac{\mu P(D-2 t)}{2 t},
$$

where $\mu$ is Poisson's ratio of the pipeline.

3.2. Stress Induced by the Temperature Differential. American Lifelines Alliance [18] proposed the equation to obtain the stress of the pipeline induced by the temperature differential:

$$
\sigma_{\mathrm{t}}=E \lambda\left(T-T_{0}\right)-\nu \sigma_{h},
$$

where $E$ is Young's modulus of the pipeline, $\lambda$ is the coefficient of thermal expansion, $T$ is the current temperature of the pipeline, $T_{0}$ is the temperature of the pipeline during pipeline installation, $v$ is Poisson's ratio for the pipeline material, and $\sigma_{h}$ is the hoop stress due to internal pressure.

3.3. Stress Induced by the Pipeline Bending. It is obvious that the pipeline will deform and bend under soil collapse. Therefore, the axial stress of the pipeline will be induced. The maximum stress $\sigma_{\mathrm{b}}$ around the circumference of the pipeline at a certain point is

$$
\sigma_{\mathrm{b}}=\frac{M(x) D}{2 I}
$$

Substituting the moment obtained by using Equations (22)-(24) into Equation (34), the axial stress can be calculated under various $\rho$ values.

3.4. Total Stress of the Pipeline. The axial stress $\sigma_{\mathrm{a}}$, hoop stress $\sigma_{\mathrm{c}}$, and radial stress $\sigma_{\mathrm{r}}$ can be obtained by the principle of superposition:

$$
\left.\begin{array}{l}
\sigma_{\mathrm{a}}=\sigma_{\mathrm{b}}+\sigma_{\mathrm{t}}+\sigma_{\mathrm{pa}}, \\
\sigma_{\mathrm{c}}=\sigma_{\mathrm{pt}}=\frac{P(D-2 t)}{2 t}, \\
\sigma_{\mathrm{r}}=-P .
\end{array}\right\}
$$

Therefore, the Mises stress can be calculated by

$$
\sigma=\sqrt{\frac{1}{2}\left[\left(\sigma_{\mathrm{a}}-\sigma_{\mathrm{c}}\right)^{2}+\left(\sigma_{\mathrm{a}}-\sigma_{\mathrm{r}}\right)^{2}+\left(\sigma_{\mathrm{c}}-\sigma_{\mathrm{r}}\right)^{2}\right]} .
$$

For the sake of the normal operation of the pipeline,

$$
\sigma \leq \sigma_{\mathrm{s}}
$$

where $\sigma_{\mathrm{s}}$ is the yield strength of the pipeline.

\section{Validation of the Calculation Model}

4.1. Reduction of the Model. The Pasternak model introduced a parameter which presents the shear interaction between adjacent soil spring elements to overcome the shortcomings of the Winkle model. In this study, the three-dimensional effect of the beam width on the behavior of the pipeline is also considered. However, when the shear modulus of the soil $G_{P}$ equals 0 and the three-dimensional effect of the beam width is also neglected, the Pasternak model reduces to the Winkle model.

4.2. Calculation Case. Test results of the PE 100 pipeline proposed by Chen [19] are used to validate the proposed method. The outer diameter of the pipeline equals $0.09 \mathrm{~m}$, and the thickness of the pipeline is $0.0043 \mathrm{~m}$. Young's modulus of the pipeline is $1.0 \mathrm{GPa}$. The pipeline is buried in sand, and the buried depth is $2 \mathrm{~m}$. The parameters of the sand shown in Table 1 are obtained using the methods 


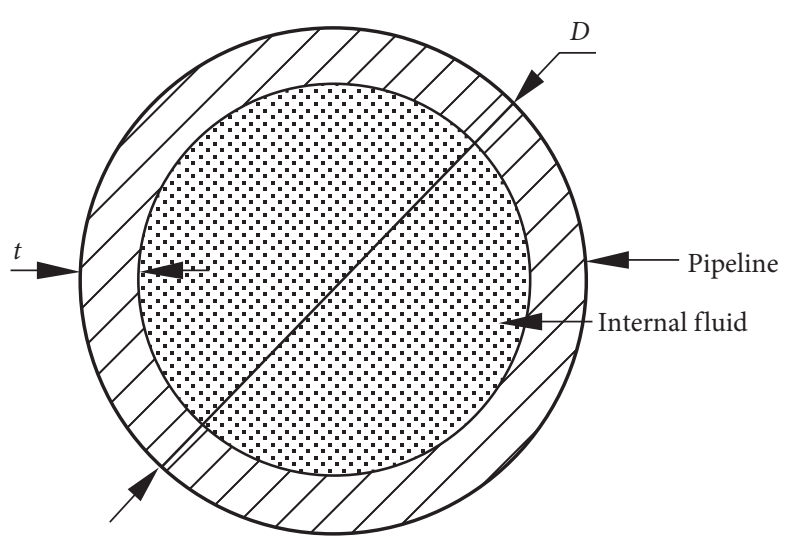

(a)

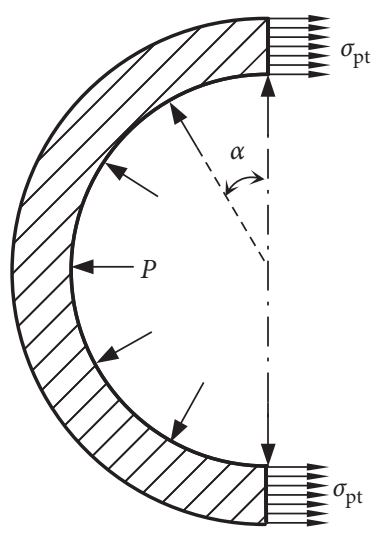

(b)

FIgURE 3: Tangential stress of the pipeline induced by internal pressure.

TABLE 1: Calculation parameters.

\begin{tabular}{lcc}
\hline$k\left(\mathrm{MN} \cdot \mathrm{m}^{-3}\right)$ & $G_{p}\left(\mathrm{MN} \cdot \mathrm{m}^{-1}\right)$ & $q\left(\mathrm{kN} \cdot \mathrm{m}^{-2}\right)$ \\
\hline 30.3 & 4.15 & 32.5 \\
\hline
\end{tabular}

proposed by Tanahashi [14]. The width of soil caves is $0.8 \mathrm{~m}$, $1.0 \mathrm{~m}$, and $1.2 \mathrm{~m}$. Table 2 shows displacements obtained from model tests by using the Winkler and the Pasternak models.

As illustrated by Chen [19], a considerable amount of the stress of the pipeline is induced by the pipeline bending. The maximum axial stresses of the pipeline caused by pipeline bending for the soil cave widths of $0.8 \mathrm{~m}, 1.0 \mathrm{~m}$, and $1.2 \mathrm{~m}$ are 14.3 $\mathrm{MPa}, 20.6 \mathrm{MPa}$, and 27.9 $\mathrm{MPa}$, respectively. Since the tensile strength of the PE 100 pipeline ranges from $20 \mathrm{MPa}$ to $25 \mathrm{MPa}$, plastic failure occurs for the pipeline when the soil cave width equals $1.2 \mathrm{~m}$. On the contrary, when the width of the soil cave equals $0.8 \mathrm{~m}$ and $1.0 \mathrm{~m}$, elastic behavior occurs for the pipelines, and the vertical displacement calculated by using the Pasternak model in the case of $\rho<1$ is larger than that by using the Winkler model. Therefore, when the axial stress of the pipeline approaches the yield stress, the Pasternak model can predict vertical displacement with higher accuracy compared with the Winkler model.

\section{Influence Factors of the Maximum Vertical Displacement and Stress}

In this section, the influence of the pipeline outer diameter, the pipeline buried depth, the soil cave width, and Young's modulus of the pipeline on the maximum vertical displacement and stress is investigated. The coefficient of the subgrade reaction is $28.3 \mathrm{MN} \cdot \mathrm{m}^{-3}$, and the shear modulus of the soil is $4.25 \mathrm{MN} \cdot \mathrm{m}^{-1}$. In addition, the outer diameters of the pipeline are $0.28 \mathrm{~m}, 0.315 \mathrm{~m}, 0.355 \mathrm{~m}, 0.4 \mathrm{~m}$, and $0.5 \mathrm{~m}$, respectively. In this study, the maximum settlement ratio $\eta$ and the maximum stress ratio $\varphi$ are defined to show the deformation and stress variations. $\eta$ and $\varphi$ can be expressed as

$$
\eta=\frac{S_{\max }}{D}
$$

where $S_{\max }$ and $D$ are the maximum settlement and the outer diameter of the pipeline.

$$
\varphi=\frac{\sigma_{\max }}{\sigma_{\mathrm{s}}}
$$

where $\sigma_{\max }$ and $\sigma_{\mathrm{s}}$ are the maximum stress and the yield stress of the pipeline.

5.1. Influence of the Pipeline Outer Diameter. Figures 4 and 5 show the responses of the maximum settlement ratio and the maximum stress ratio to the pipeline dimension. The pipeline dimension is expressed in the normalized form of $D / t$, where $t$ is the pipeline thickness. It can be concluded from Figure 4 that the $\eta$ value follows a linear relationship with the normalized pipeline dimension. The main reason is that the flexural rigidity of the pipeline decreases with increasing $D / t$.

As shown in Figure 5, the maximum stress ratio also increases with increasing $D / t$. The main reason is that the inertia moment of the pipeline decreases with the increasing $D / t$ resulting in the increase in the stress.

5.2. Influence of the Buried Depth. Figures 6 and 7 give the maximum settlement ratio and the maximum stress ratio to the pipeline under various pipeline buried depths. The pipeline buried depth is expressed in the normalized form of $H / D$, where $H$ is the buried depth. It can be concluded that the $\eta$ and $\varphi$ values increase linearly with the increasing pipeline buried depth.

5.3. Influence of the Soil Cave Width. The variations of the maximum settlement ratio and the maximum stress ratio with the normalized soil cave width $L / D$ are given in Figures 8 and 9 , where $L$ is the soil cave width. As shown in Figure 8, the maximum settlement ratio increases nonlinearly with the increase of the soil cave width. The main reason is that the length of the suspended pipeline increases with the increasing soil cave width, resulting in the rapid increase in both the displacement and the stress of the pipeline. According to Figures 8 and 9, it can be concluded that the soil cave width governs the pipeline stability. 
TABLe 2: Calculated vertical displacements.

\begin{tabular}{lccc}
\hline Width of the soil cave $(\mathrm{m})$ & Test results, $Y_{0}(\mathrm{~mm})$ & Winkler model, $Y_{1}(\mathrm{~mm})$ & Pasternak model, $Y_{2}(\mathrm{~mm})$ \\
\hline 0.8 & 17.9 & 16.4 & 22.8 \\
1.0 & 49.7 & 29.1 & 43.2 \\
1.2 & 79.4 & 48.7 & 73.9 \\
\hline
\end{tabular}

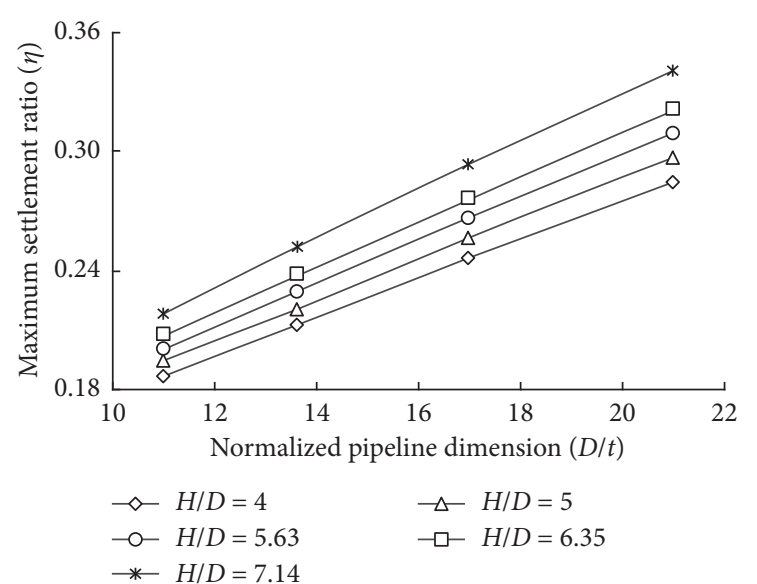

FIgURE 4: Influence of pipeline dimension on the maximum settlement ratio.

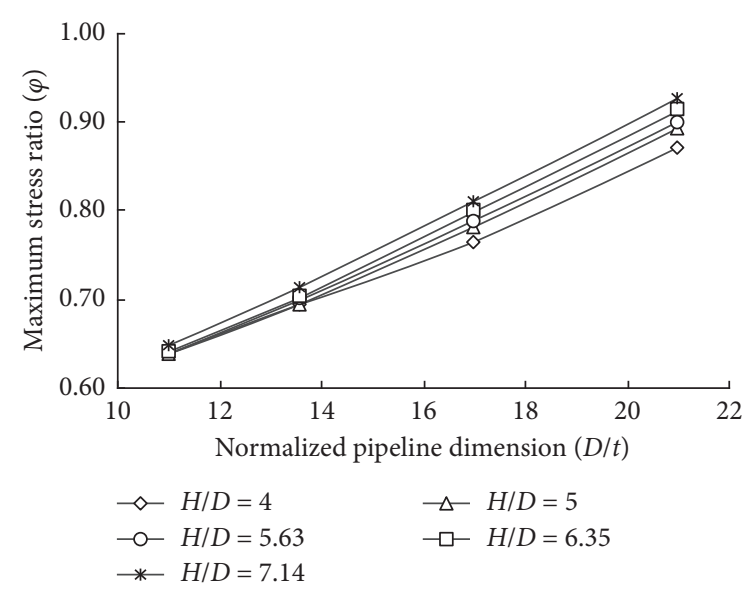

FIGURE 5: Influence of pipeline dimension on the maximum stress ratio.

5.4. Influence of Young's Modulus of Pipeline. Figures 10 and 11 show the influences of the pipeline Young's modulus on the maximum settlement ratio and the maximum stress ratio. The vertical displacement decreases with the increasing Young's modulus of the pipeline. However, Young's modulus has little effect on the maximum stress of the pipeline.

\section{Conclusions}

The main conclusions can be summarized as follows:

(1) A new solution for calculating the deflection and internal force of the pipeline was proposed based on Pasternak's beam-on-elastic-foundation theory.

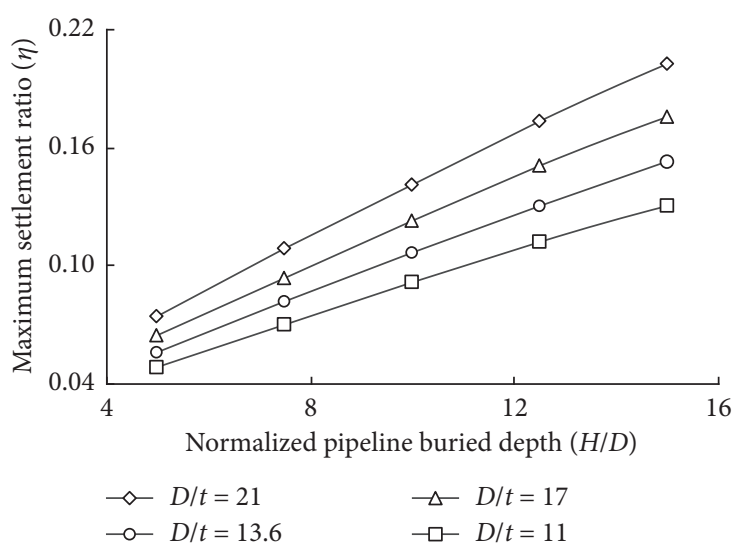

FIGURE 6: Influence of pipeline buried depth on the maximum settlement ratio.

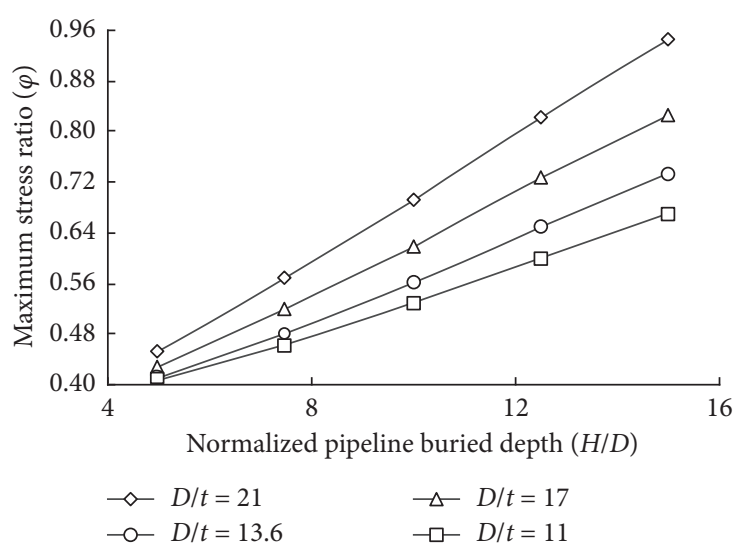

Figure 7: Influence of pipeline buried depth on the maximum stress ratio.

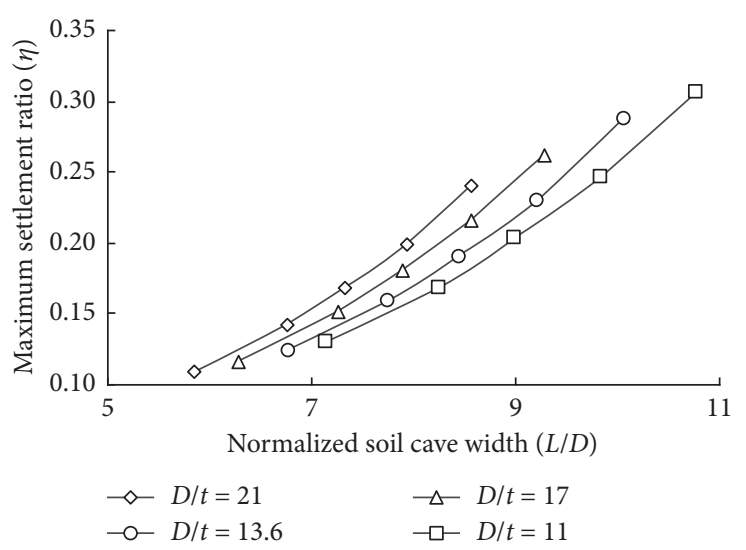

FIGURE 8: Influence of soil cave width on the maximum settlement ratio. 


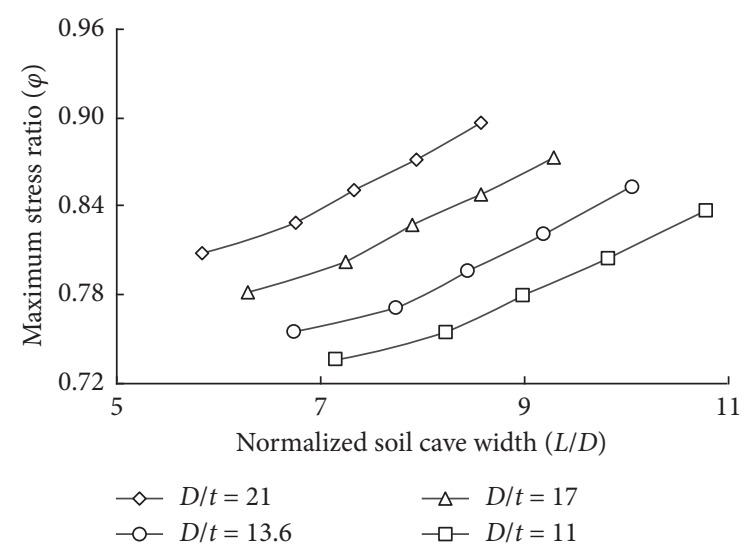

FIGURE 9: Influence of soil cave width on the maximum stress ratio.

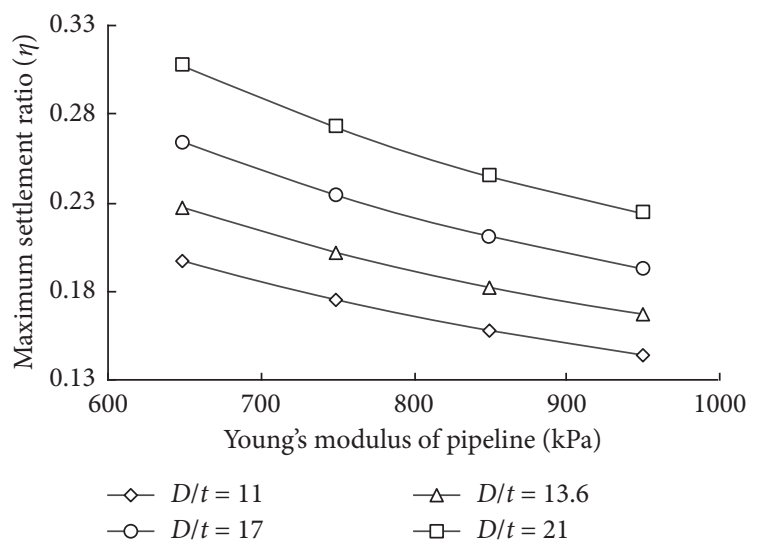

FIgURE 10: Influence of pipeline Young's modulus on the maximum settlement ratio.

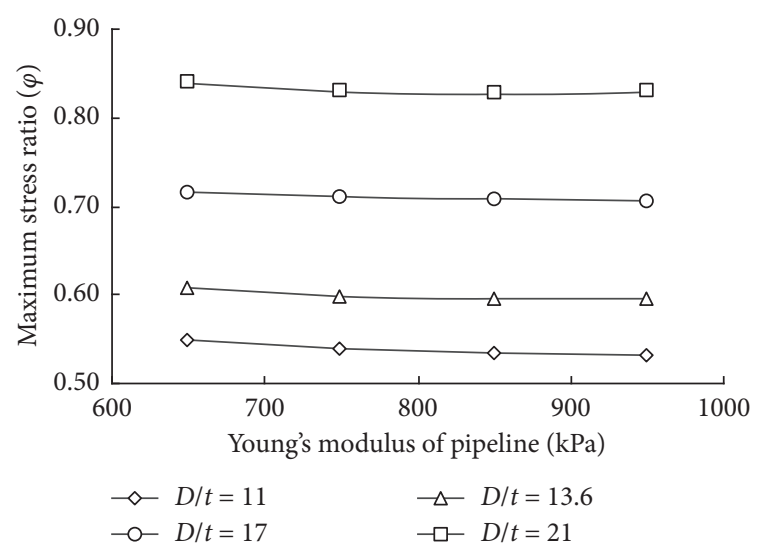

FIgURE 11: Influence of pipeline Young's modulus on the maximum stress ratio.

Compared with the previous studies, the proposed mechanical model employed the Pasternak model, which considered the continuity problem in the foundation soil and the influence of the foundation beam width on the pipe deformation. So it is closer to the practical working condition.
(2) The proposed solution can provide calculated results with higher accuracy compared with the Winkler model, especially when the axial stress of the pipeline approaches the yield stress, which means that the proposed method can well predict the behavior of the pipeline in the limit state.

(3) The parameter $\rho$ in model can be divided into three cases: $\rho<1, \rho=1$, and $\rho>1$. These three cases are solved separately, and all situations involved in the Pasternak model are calculated theoretically, which correspond to various conditions in practical engineering.

(4) The analysis of the stress state of the pipeline under the collapse effect provides a basis for judging whether the pipeline is failure. Meanwhile, the applicable scope of the Pasternak model to calculate the deformation and internal forces of the pipeline is clarified.

\section{Data Availability}

The data used to support the findings of this study are available from the corresponding author upon request.

\section{Conflicts of Interest}

The authors declare that they have no conflicts of interest.

\section{References}

[1] K. Tanabe and S. Takada, "Design formulae of buried pipes subject to large ground settlement and its application," Doboku Gakkai Ronbunshu, no. 374, pp. 593-602, 1986.

[2] H. Gao, "Response analysis for buried pipelines through settlement zone," Earthquake Engineering \& Engineering Vibration, vol. 17, no. 1, pp. 68-75, 1997.

[3] Q. Chen and W. Zhang, "Compressor unit configuration for the compressor stations of west-to-east gas pipeline project," Oil \& Gas Storage and Transportation, vol. 23, no. 4, pp. 6-8, 2004.

[4] X. L. Wang and A. L. Yao, "Deflection and internalforce analysis of buried steel pipelines in partial hanging," Engineering Mechanics, vol. 25, no. 8, pp. 218-222, 2008.

[5] G. P. Kouretzis, D. K. Karamitros, and S. W. Sloan, "Analysis of buried pipelines subjected to ground surface settlement and heave," Canadian Geotechnical Journal, vol. 52, no. 8, pp. 1058-1071, 2014.

[6] J. Ma, J. Peng, X. Gao, and L. Xie, "Effect of soil-structure interaction on the nonlinear response of an inextensional beam on elastic foundation," Archive of Applied Mechanics, vol. 85, no. 2, pp. 273-285, 2015.

[7] Z. Cao, J. Han, C. Xu, D. K. Khatri, R. Corey, and Y. Cai, "Road surface permanent deformations with a shallowly buried steel-reinforced high-density polyethylene pipe under cyclic loading," Geotextiles and Geomembranes, vol. 44, no. 1, pp. 28-38, 2016.

[8] X. Luo, S. Lu, J. Shi, X. Li, and J. Zheng, "Numerical simulation of strength failure of buried polyethylene pipe under foundation settlement," Engineering Failure Analysis, vol. 48, pp. 144-152, 2015.

[9] H.-S. Kim, W.-S. Kim, I.-W. Bang, and K. H. Oh, "Analysis of stresses on buried natural gas pipeline subjected to ground 
subsidence," in Proceedings of the 2nd International Pipeline Conference, pp. 749-756, American Society of Mechanical Engineers, Calgary, Canada, 1998.

[10] J. Yu, C. Zhang, and M. Huang, "Soil-pipe interaction due to tunnelling: assessment of Winkler modulus for underground pipelines," Computers and Geotechnics, vol. 50, pp. 17-28, 2013.

[11] S. Iimura, "Simplified mechanical model for evaluating stress in pipeline subject to settlement," Construction and Building Materials, vol. 18, no. 6, pp. 469-479, 2004.

[12] S. S. Peng and Y. Luo, "Determination of stress field in buried thin pipelines resulting from ground subsidence due to longwall mining," Mining Science and Technology, vol. 6, no. 2, pp. 205-216, 1988.

[13] Y. Luo, S. Peng, and H. Chen, "Protection of pipelines affected by surface subsidence," Transactions, vol. 302, pp. 98-103, 1997.

[14] H. Tanahashi, "Formulas for an infinitely long Bernoulli-Euler beam on the Pasternak model," Soils and Foundations, vol. 44, no. 5, pp. 109-118, 2004.

[15] P. L. Pasternak, On a New Method of an Elastic Foundation by Means of Two Foundation Constants, State Publishing House of Literature in Construction and Architecture, Moscow, Russia, 1954.

[16] A. P. S. Selvadurai and G. M. L. Gladwell, "Elastic analysis of soil-foundation interaction," Journal of Applied Mechanics, vol. 47, no. 1, p. 219, 1980.

[17] C. P. Press, Code for Design of Gas Transmission Pipeline, Beijing, China, 2015.

[18] A. L. Alliance, Guidelines for the Design of Buried Steel Pipe, ASCE, Reston, VA, USA, 2001.

[19] Z. L. Chen, Experimental Research and Analysis of the Pipeline Deformation Mechanism Caused by Subsoil Settlement, Taiyuan University of Technology, Taiyuan, China, 2013. 


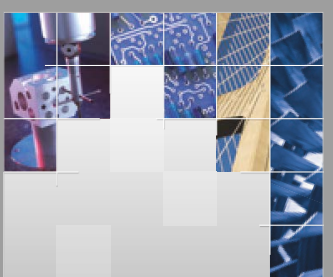

\section{Enfincering}
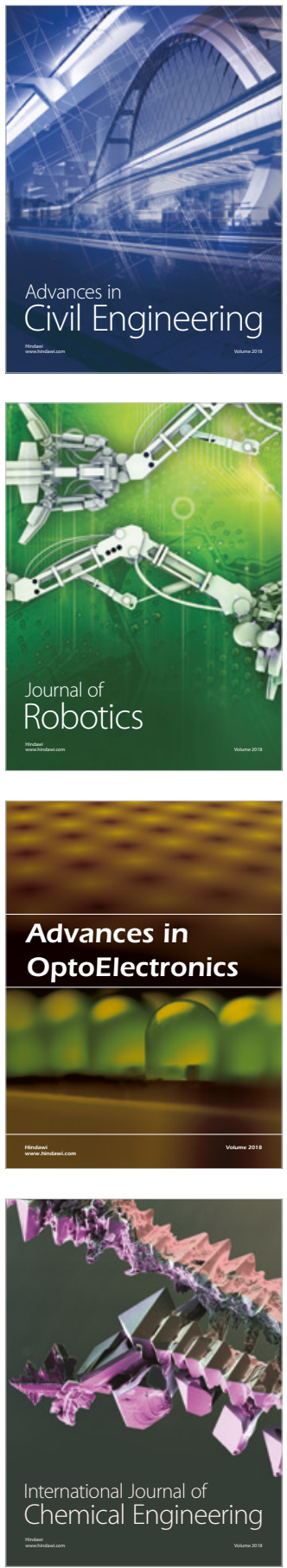

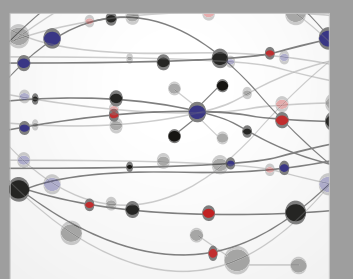

\section{Rotating \\ Machinery}

The Scientific World Journal

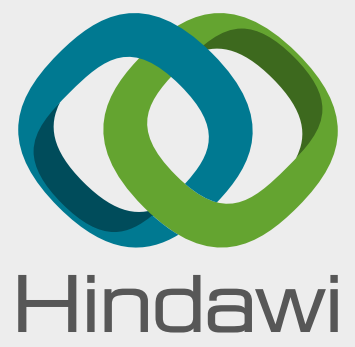

Submit your manuscripts at

www.hindawi.com
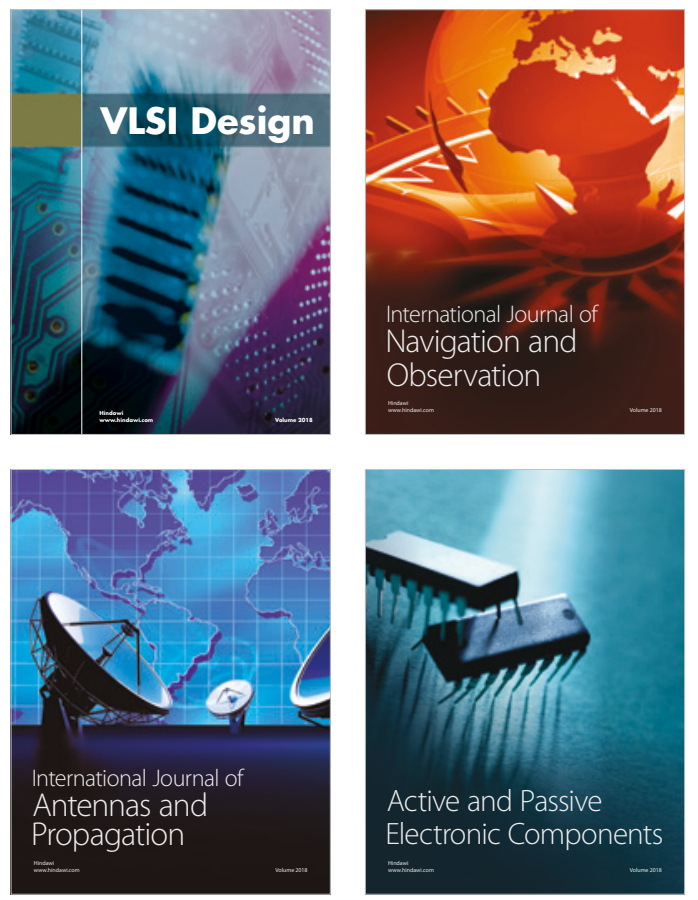
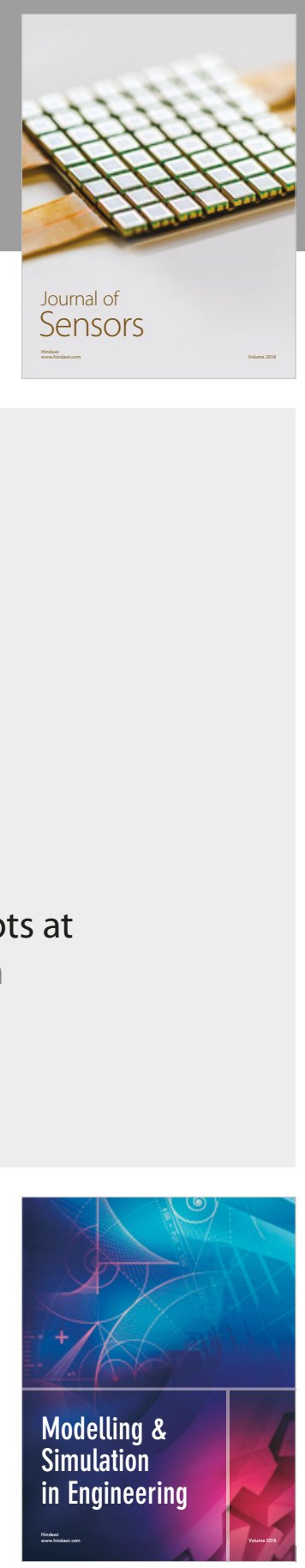

\section{Advances \\ Multimedia}
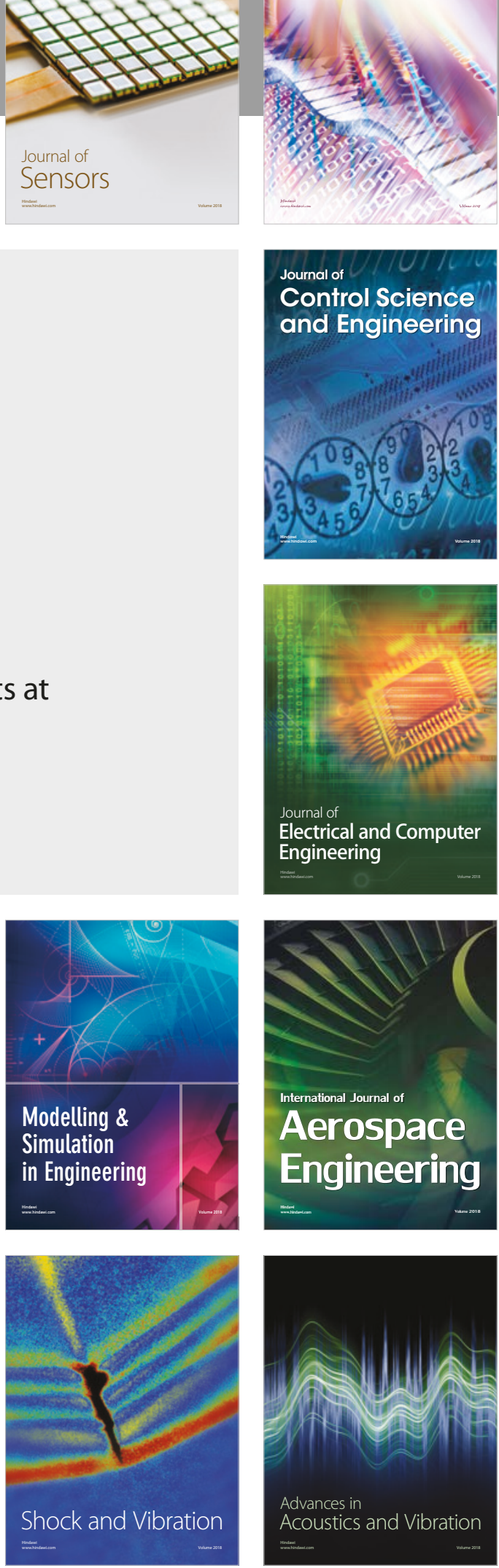